\title{
Performance Analysis and Enhancement of the Next Generation Cellular Networks
}

\author{
Xiang Yu ${ }^{\dagger}$, Chunming Qiao*, Xin Wang ${ }^{\ddagger}$ and Dahai $\mathrm{Xu}^{\diamond}$ \\ ${ }^{\dagger}$ Department of Computer Science, Frostburg State University \\ *Department of Computer Science and Engineering, State University of New York at Buffalo \\ ${ }^{\ddagger}$ Department of Electrical Engineering, State University of New York at Stony Brook \\ ${ }^{\diamond}$ Department of Electrical Engineering, Princeton University
}

\begin{abstract}
As more and more wireless subscribers access the Internet through cellular networks, Internet data traffic, which is known to be long range dependent (LRD), will soon dominate the conventional voice traffic. In this paper, we study the impact of such LRD data traffic on the statistical characteristics of Multi-Access Interference (MAI) and Signal to Interference-plus-Noise Ratio (SINR) in a Code Division Multiple Access (CDMA) network. Through analysis and simulation, we show that the timescaled MAI and SINR have slow decaying tail distributions due to the LRD data traffic. As a result, the outage probability is larger for data users than that for voice users.

To improve the performance of the CDMA network in the presence of LRD data traffic, we propose a variable period prediction scheme to predict MAI or the equivalent number of active users. We show that the proposed variable period prediction is not only more accurate for data users but also less memory-consuming than existing fixed period prediction. In addition, rate control and call admission control based on variable period prediction can achieve lower outage probability and higher throughput for data users than that based on fixed period prediction.
\end{abstract}

\section{INTRODUCTION}

The first generation and most of the second generation cellular systems are developed mainly for voice services as most of the subscribers are voice users. However, in the coming third generation mobile systems, most subscribers will access the Internet through the wireless cellular networks for email, file transfer, web browsing, multimedia and other Internet applications. As new Internet based services and applications continue to emerge, it is expected that the traffic of the next generation mobile cellular networks will be dominated by the Internet data.

Understanding the nature of the traffic in the next generation mobile networks is critical for efficient network protocol and system design. Since Code Division

This work is partially supported by NSF grant 0435155 , NSF CNS0448405 and OJP 2004-LT-BX-K027
Multiple Access (CDMA) [1] is currently the dominant technology for wireless cellular networks in North America, and expected to continue to play an important role in the next generation cellular networks, we are particularly interested in the performance impact of Internet data traffic on a CDMA network.

In a CDMA network, traffic transmissions by all other active users contribute to Multi-Access Interference (MAI) of an individual user. Therefore, the characteristics of the aggregated traffic transmitted by all other users affect the characteristics of MAI, and in turn Signal to Interference Noise Ratio (or SINR) which indicates the quality of the received signal of the individual user. While the conventional voice traffic in a CDMA network is usually modeled as Poisson process with an exponential interarrival time of packets or bursts, with the introduction of Internet applications mentioned above, Poisson processes can no longer characterize the aggregated data traffic transmitted over a CDMA network. More specifically, it has been shown in [2] that the Internet traffic is much more bursty over larger time scales than voice traffic, and is usually characterized as a self-similar or Long Range Dependent (LRD) process. In this paper, we use the Weibull Bounded Burstiness (WBB) process in [3] to characterize the LRD characteristics in a CDMA network and study the impact of long range dependency on MAI as well as SINR in a CDMA network with many data users.

Most of traditional performance studies in CDMA networks have been focused on the (long term) average MAI and SINR. The admission control mechanisms and most of the other related techniques developed in CDMA networks are based on these average performance measures of the system, which works well when voice traffic dominates. However, with data traffic which is more bursty and exhibits long range dependency, one needs to look beyond the average performance measures and instead, focuses more closely on the correlated behavior.

The need for looking beyond the average behavior is 
that a system with many data users may experience, for example, a longer period of having a large number of active users followed by a longer period of having a small number of users than a system with voice users, even though the two systems may have the same average number of users. In other words, in the system with data users, the distribution and autocorrelation of both the MAI and SINR will be different from those in a system with only voice users. Such differences will lead to different outage probabilities and throughputs, and also affect other mechanisms including rate control and Call Admission Control (CAC) in the two systems.

So far, there have been only limited studies on the distribution and autocorrelation of the MAI and SINR in a CDMA system with data users. For example, it has been first proved in [4], [5] that LRD Internet traffic transmitted by a large number of data users in a CDMA system results in LRD MAI. However, [4], [5] only studied MAI's self-similarity, without giving any analytical distribution of MAI and other performance measures including SINR and outage probability.

In this paper, starting from the impact of LRD data traffic on MAI, the statistical characteristics and the tail distribution of MAI (or equivalently the number of active users) and SINR in the presence of many data users are analyzed. One of our major contributions is the development of statistical formulas for both MAI and SINR distribution and tail probability in different time scales. It is found through both analysis and simulation that both MAI and SINR have slow decaying tails in large time scales, and in addition, the corresponding outage probability is also higher due to the presence of LRD data traffic. Another major contribution of this paper is that a Variable Period (VP) prediction scheme taking into consideration such impact is proposed and demonstrated to outperform Fixed Period (FP) prediction proposed in [4], [5]. We show that the proposed VP prediction is not only more accurate for data users but also less memoryconsuming than FP prediction. The VP prediction is applied to rate control and $\mathrm{CAC}$, and our simulation results show that both rate control and CAC based on VP prediction achieve higher throughput and lower outage probability than those based on FP prediction or those without prediction at all. Our studies further verify the usefulness of the proposed VP prediction in supporting data users in future CDMA networks.

The rest of this paper is organized as follows. Section II introduces the basic concept related to LRD processes. Section III studies the impact of long range dependency on MAI, SINR and outage probability in CDMA networks, via both analysis and simulation. To enhance the network performance for data users, a
Variable Period (VP) prediction scheme is proposed in Section IV to predict MAI and the number of active users in the CDMA system. Its performance is compared with that of Fixed Period (FP) prediction in terms of prediction accuracy. In Sections V and VI, VP prediction is applied to rate control and Call Admission Control (CAC), which achieve higher throughput and lower outage probability in the CDMA network for data users than the existing rate control and CAC based on FP prediction or those without prediction. Finally, Section VII concludes this paper.

\section{Long Range Dependent Process}

In this section, we introduce the concept of long range dependent (LRD) processes, which is useful in understanding the discussion and especially the formulas in the subsequent sections.

A LRD process is often characterized by heavy traffic bursts that extend over a wide range of time scales [2], [6]. Suppose $A$ is a discrete LRD process, and $A(u)$ denotes the $u$-th sampling of $A$. Define $A^{T}$ to be the average of $A$ aggregated in a time interval $T$. The $v$-th sampling of $A^{T}$ is expressed as

$$
A^{T}(v)=\frac{1}{S_{T}} \sum_{u=(v-1) S_{T}+1}^{v S_{T}} A(u),
$$

where $S_{T}$ is the total number of samplings contained in $T$. We call $A$ as the instantaneous process, and accordingly $A^{T}$ is called the time-scaled process of $A$.

Denote the autocorrelation of $A$ by $r_{A}(m)$ (which is defined to be $E[A(u) A(u+m)])$, and the autocorrelation of $A^{T}$ by $r_{A}^{T}(m)$. A Short Range Dependent (SRD) process has $r_{A}^{T}(m)<r_{A}(m)$ and $r_{A}^{T}(m)$ decreases with $T$. However, for an LRD process, $r_{A}^{T}(m)=r_{A}(m)$ for any $m$ with $T \rightarrow \infty$, or in other words, the autocorrelation of time-scaled process is similar to that of the instantaneous process [7]. The variance of time-scaled process also exhibits similar trend, i.e., it decreases much faster with $T$ for an SRD process than that for an LRD process [8].

A time-scaled LRD process $A^{T}$ often possesses a tail distribution that decays slower than that of timescaled SRD process. It has been shown that the tail distribution of $A^{T}$ has a Weibull bound, which is generally larger than the exponential bound associated with an SRD process. Accordingly, $A$ can be modelled as a discrete Weibull Bounded Burstiness (WBB) process [3] as $\operatorname{WBB}(C, \mu, \nu)$ with parameters $C>0$ (which denotes the asymptotic constant), $\mu>0$ (which denotes the decay rate), and $0<\nu=2-2 H \leq 1$ (which denotes 
the index parameter) ${ }^{1}$, and the tail distribution of $A^{T}$ satisfies a Weibull bound as follows:

$$
\operatorname{Pr}\left\{A^{T}(v)>E[A]+x\right\} \sim C e^{-\mu\left(x S_{T}\right)^{\nu}}
$$

for all $x \geq 0$, all $T>0$ and all $v \geq 0$, where $E[A]$ is the long term average value of $A$ and $x$ is defined as the tail of $A^{T}(v)$.

\section{IMPACT OF LRD ON A CDMA SYSTEM}

In this section, we study the impact of LRD data traffic on a CDMA network. In particular, we consider the impact of LRD data traffic on uplink performance of a CDMA system, and show through analysis and simulation that both MAI and SINR have slow decaying tails in large time scales, and in addition, the corresponding outage probability is also higher due to the presence of LRD data traffic.

Suppose there are $N$ users in the CDMA network and let $X_{i}(u)$ be the activity indicator of user $i$ at the $u$ th sampling time. $X_{i}(u)$ is an ON/OFF process. During the ON period, $X_{i}(u)=1$ and the user transmits at a constant rate $R_{i}$ with a transmission power $P_{i}$ (per time unit), while during the OFF period, $X_{i}(u)=0$ and the user does not transmit, i.e.,

$$
X_{i}(u)=\left\{\begin{array}{l}
1, \quad \text { if user } i \text { is } O N \\
0, \quad \text { if user } i \text { is } O F F
\end{array}\right.
$$

Note that $R_{i} X_{i}$ is the traffic arrival process of user $i$. Since the inter-arrival time of data bursts for Internet data users could be either lognormal or Pareto distributed [2], it indicates that the distribution of OFF period duration $T_{\text {off }}$ is usually heavy tailed as:

$$
P\left\{T_{\text {off }}>x\right\} \sim x^{-\alpha}
$$

for any given $x>0$. Here $\alpha=3-2 H$ and $1<$ $\alpha \leq 2$. Such an ON/OFF process is also called a heavy tailed ON/OFF process. It has been demonstrated that the aggregation of multiple such heavy tailed ON/OFF processes, i.e., $\sum_{j=1}^{N} X_{j}(u)$, is an LRD process [7], [9], [10].

\section{A. LRD Impact on MAI}

In this subsection, we first develop formulas to show that a time-scaled MAI has a slow decaying tail distribution and then verify this claim through simulation results.

${ }^{1}$ Here $0.5 \leq H<1$ is the Hurst parameter indicating the degree of LRD, and a larger $H$ is usually associated with a heavier LRD process. In particular, an SRD flow is always associated with $H=0.5$ or $\nu=1$
We assume that the CDMA system implements power control [11], [12] to achieve the same Signal to Interference Ratio (SIR) at the base station for every user so that no user gains better performance with a transmission power higher than necessary. Suppose $P_{j}$ is the receiving power per time unit detected at the base station for user $j$ who is currently transmitting. With power control, the receiving power per bit $P_{j} / R_{j}$ from any user $j$ is the same, which equals to a constant power $P$ (per data bit), i.e., $P_{j} / R_{j}=P$.

The MAI detected by an individual user $i$ at the $u$-th sampling time is calculated as the sum of the receiving power $P_{j}$ of all the other existing users in the system except for user $i$ itself, i.e., the $u$-th sampling of MAI is expressed as

$$
I_{i}(u)=\Sigma_{j=1, j \neq i}^{N} P_{j} X_{j}(u)
$$

With power control, $P_{i} / R_{i}=P_{j} / R_{j}$, and Eq. (4) is rewritten as

$$
I_{i}(u)=P_{i} \Sigma_{j=1, j \neq i}^{N} X_{j}(u) \frac{R_{j}}{R_{i}}=P_{i} K_{i}(u),
$$

where $K_{i}(u)=\sum_{j=1, j \neq i}^{N} X_{j}(u) \frac{R_{j}}{R_{i}}$ is the equivalent number of active users (seen by user $i$ ) who transmit with the same data rate $R_{i}$ as user $i$. In particular, when all users transmit at the same data rate, i.e., $R_{i}=R_{j}$, then $K_{i}(u)$ is the actual number of active users in the system. For brevity, we call $K_{i}$ simply "the number of active users" (i.e., we will not use the word "equivalent").

Since $X_{j}(u)$ is a heavy tailed ON/OFF process, $X_{j}(u) \frac{R_{j}}{R_{i}}$ is still a heavy tailed ON/OFF process. Therefore, the aggregated process $K(u)$ of such multiple heavy tailed ON/OFF processes is an LRD process. Accordingly, the autocorrelation of $K$ and $K^{T}$ are identical, i.e., $r_{K}^{T}(m)=r_{K}(m)$. Since MAI $I_{i}(u)=P_{i} K(u)$, where $P_{i}$ is a constant, $I_{i}(u)$ is also an LRD process. A similar proof that shows MAI is LRD can be found in [4], [5]. In this paper, we model MAI with a discrete WBB process, and the time-scaled MAI $I_{i}^{T}$ has a Weibull bound on its tail distribution according to Eq. (2):

$$
\operatorname{Pr}\left\{I_{i}^{T}(v)>E\left[I_{i}\right]+x\right\} \sim C e^{-\mu\left(x S_{T}\right)^{\nu}}
$$

for any $v>0$. Here $E\left[I_{i}\right]$ is the long term average MAI and $x$ is the tail of the $I_{i}^{T}$. From Eq. (6) we can see that increasing time scale $T$ (or $S_{T}$ ) decreases the tail probability. In particular, since data users are associated with $\nu<1$ while voice users are associated with $\nu=1$, the tail probability for voice users decreases with $T$ faster than that for data users.

The Weibull bound only characterizes the tail distribution of time-scaled MAI, which is not sufficient to characterize the general distribution of $I_{i}^{T}$. Since the user 
behavior is independent of each other, the instantaneous MAI $I_{i}$ is the sum of $P_{j} X_{j}$, which follows a Gaussian distribution according to the central limit theorem. Suppose $I_{i}$ has a mean $P_{i} E[K]$ and a variance $P_{i}^{2} \sigma_{K}^{2}$. For voice users, MAI is an SRD process where the central limit theorem also applies to the time-scaled process beyond some small $T$. Hence, $I_{i}^{T}$ still follows a Gaussian distribution with mean $P_{i} E[K]$ and variance $P_{i}^{2} \sigma_{K}^{2} / S_{T}$ as predicted by the central limit theorem. However, for data users, MAI is an LRD process and the central limit theorem does not apply. Instead, $I_{i}^{T}$ approximates a Gaussian distribution with the same mean $P_{i} E[K]$ but a larger variance $P_{i}^{2} \sigma_{K}^{2} / S_{T}^{2-2 H}$ given that $0.5<H<1$ [7], [8]. In other words, time-scaled process $I_{i}^{T}$ has a variance decreasing slower with $T$, with a PDF

$$
f_{I_{i}^{T}}(x)=\frac{1}{\sqrt{2 \pi P_{i}^{2} \sigma_{K}^{2} / S_{T}^{2-2 H}}} e^{-\frac{\left(x-P_{i} E\left[K_{i}\right]\right)^{2}}{2 P_{i}^{2} \sigma_{K}^{2} / S_{T} T^{2-2 H}}}
$$

Examples of such Gaussian LRD processes include fractional Brownian motion and fractional Gaussian noise (FGN) processes in [13]. Note that, MAI may not be approximated with a Gaussian process because of channel fading [5]. (However, as power control is normally performed in CDMA networks, the effect of channel fading can be hided and the receiving power is roughly the same for all the users. Therefore, such an approximation is still valid.)

\section{Simulation Results}

To verify that the time-scaled MAI in a system with LRD data traffic does have a slow decaying tail distributions and in particular it approximates a Gaussian distribution with a larger variance, we simulate two CDMA systems, one with data users and the other with voice users.

We simulate 250 ON/OFF users with maximum uplink speed of $64 \mathrm{kbps}$ in both of the two CDMA systems. Suppose that each of the ON/OFF users has an average activity factor of 0.4 , i.e., the average ratio between an $\mathrm{ON}$ and an OFF period is $0.4 / 0.6$. For heavy tailed data users, we assume that the average data size transmitted during ON period is $20 \mathrm{~KB}$, which is equivalent to an $\mathrm{ON}$ period of 2.5 seconds (at $64 \mathrm{kbps}$ ); while for voice users, the average ON period is $70 \mathrm{~ms}$. Each simulation runs for 100 seconds and a sampling is taken for every bit transmitted. In the simulation, the distributions of MAI are based on total $6.4 \times 10^{6}$ samplings.

Fig. 1 illustrates the distributions of instantaneous MAI $I_{i}$ and time-scaled MAI $I_{i}^{T}$, which roughly follow the Gaussian distribution as in Eq. (7). As $T$ increases, the variance of $I_{i}^{T}$ for voice users in Fig. 1(a) decreases faster than that for data users in Fig. 1(b).
The tail distributions of $I_{i}$ and $I_{i}^{T}$ are illustrated in Fig. 2. The tail probability for data users shown in Fig. 2(b) is in general larger than that for voice users in Fig. 2(a), especially when $T$ is large as predicted by Eq. (6). In other words, $I_{i}$ for data users is an LRD process and its corresponding time-scaled process has a slower decaying tail distribution.

The above simulation results clearly back up the claim based on our analysis. In other words, they show that in a practical setting, the claim that the time-scaled MAI in a system with LRD data traffic approximates a Gaussian distribution with a slow decaying tail distributions is indeed true. However, note that we do not claim that the Eqs. (6) and (7) can accurately determine the exact tail distribution and variance due to the difficulties in obtaining the exact values of the parameters used in these equations.

\section{B. LRD Impact on SINR}

We now develop formulas to show that time-scaled SINR also has a slow decaying tail distribution, and then verify this claim using simulation results.

The SINR for an active user $i$ at the $u$-th sampling is defined as

$$
S I N R_{i}(u)=\frac{P_{i} / R_{i}}{N_{0}(u)+\frac{1}{W} \sum_{j=1, j \neq i}^{N} P_{j} X_{j}(u)},
$$

where $W$ is the spreading signal's bandwidth and $N_{0}(u)$ is the instantaneous sampling receiving power of the white Gaussian noise. In general, the SINR measured in a finite time scale $T$ is of interest for performance evaluation in a CDMA system. For voice users, SINR can be usually expressed in terms of long term average measurement of noise $E\left[N_{0}\right]$ and the number of active users $E\left[K_{i}\right][1]$ because the short term average value can be well approximated with the long term average value. However, this is not true for data users. Note that although [5] uses the instantaneous (as well as timescaled) SINR (in Eq. (8) of [5]), the Gaussian noise inside the SINR expression is still approximated with its long term average value $\sigma_{n}^{2}$, which is not accurate enough to characterize the statistics of instantaneous (as well as time-scaled) SINR.

In the appendix of this paper, we prove that the timescaled SINR has a "Gaussian-like" distribution in a large enough time scale, whose PDF can be approximated as:

$$
f_{S I N R_{i}}^{T}(z) \simeq \frac{1}{\sqrt{2 \pi \operatorname{Var}\left[h^{T}\right]|z|}} e^{-\frac{\left(\frac{1}{z}-E\left[h^{T}\right]\right)^{2}}{2 \operatorname{Var}\left[h^{T}\right]}}
$$

where $h^{T}$ is a variable decided by both time-scaled noise $N_{0}^{T}$ and time-scaled MAI (or equivalently the number 


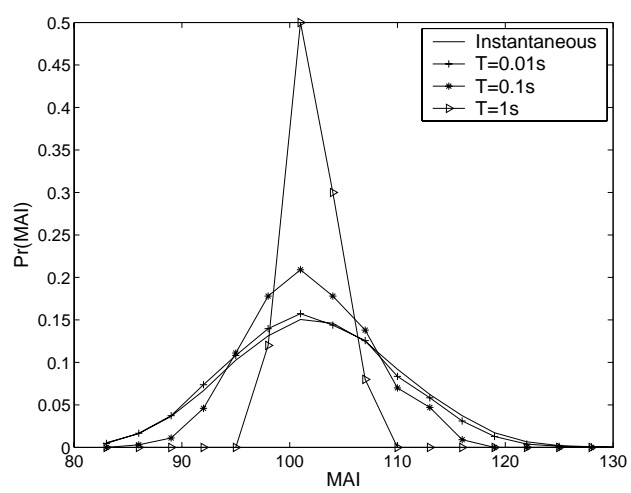

(a) voice users

Fig. 1. Distribution of MAI

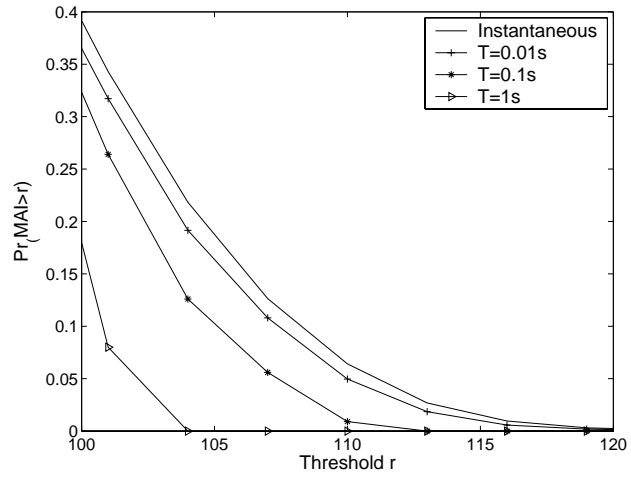

(a) voice users

Fig. 2. Tail distribution of MAI

of active users $K_{i}^{T}$ ). In particular, from the PDF of $S I N R^{T}$, we can see that the variance of $S I N R^{T}$ is related with the variance of $h^{T}$, which is consequently related with the variance of both time-scaled noise and MAI.

We also prove in the appendix of this paper that SINR has a self-similar correlation structure. Accordingly, the time-scaled SINR has a slow decaying tail distribution which is approximated as:

$\operatorname{Pr}\left\{S I N R_{i}^{T}>E\left[S I N R_{i}^{T}\right]+x\right\} \sim C e^{-\mu\left(\frac{P_{i} G_{i}}{E^{2}\left[S I N R_{i}^{T}\right]}\right)^{\nu}\left(x S_{T}\right)}$

Eq. (10) indicates that $S I N R_{i}$ is WBB process. The only difference of the WBB SINR in Eq. (10) from the WBB MAI in Eq. (6) is that the decay rate of SINR is $\left(\frac{P_{i} G_{i}}{E^{2}\left[S I N R_{i}^{T}\right]}\right)^{\nu}$ times of the decay rate of MAI.

\section{Simulation Results}

To verify the claim based on the above analysis, we use the same simulation setting as that described in Section III-A. Below, we present the simulation results for CDMA systems with low Gaussian noise only, and

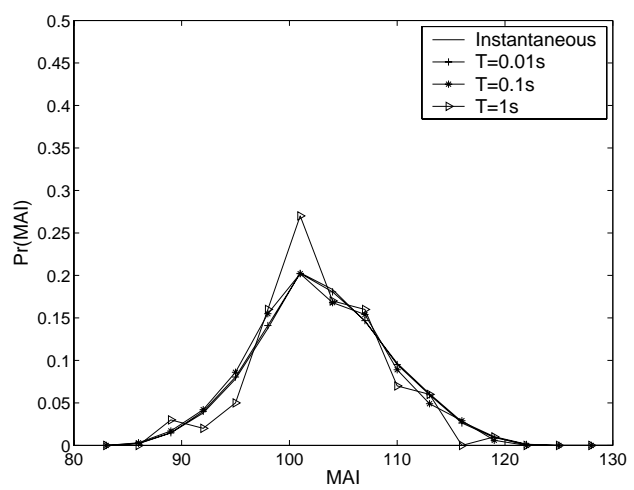

(b) data users, $H=0.9$

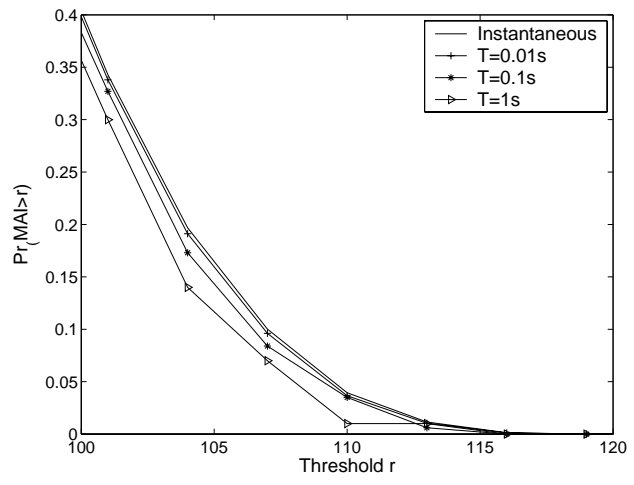

(b) data users, $H=0.9$

omit those with high Gaussian noise. The omission is due to both space limit and the fact that the results with high Gaussian noise are trivial in that both SINR for data users and voice users have similar Gaussian distributions since Gaussian noise is the dominant factor in SINR and is independent of user behaviors.

For the case where Gaussian noise is low, we set the noise $\sigma_{n}^{2}$ as only one hundredth of the signal power, i.e., $\sigma_{n}^{2}=0.01 P$ as shown in Fig. 3. In this case, MAI is the dominant factor in SINR, and the distribution of $S I N R^{T}$ is affected mostly by $I^{T}$ and its variance decreases with $T$. In particular, since the variance of $I^{T}$ for data users decreases slower with $T$ than that for voice users, the variance of $S I N R^{T}$ shows the same trend as predicted by Eq. (9).

\section{Outage Probability}

In this subsection, we show that due to the slower decaying tail distribution of time-scaled SINR for data users, the outage probability for data users will be higher than that for voice users. 


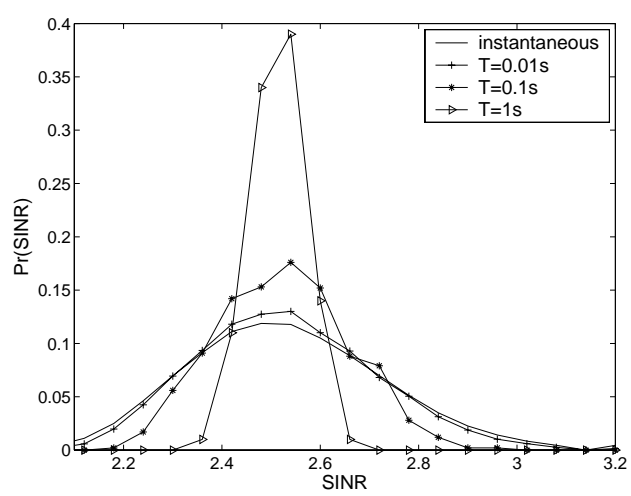

(a) voice users

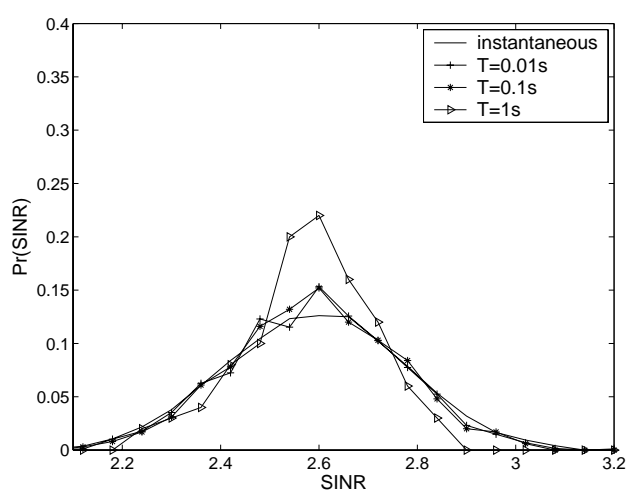

(b) data users, $H=0.9$

Fig. 3. Distribution of SINR, with low noise $\sigma_{n}^{2}=0.01 P$,

The outage probability of a CDMA system is approximated as the probability that the average SINR in a (packet) transmission period $T_{p}$ (with $S_{T_{p}}$ samplings) is smaller than a minimum threshold $S I N R_{0}=$ $E[S I N R]-x(0<x<E[S I N R])$. The outage probability is calculated as:

$$
\begin{aligned}
\operatorname{Pr}_{\text {out }} & =\operatorname{Prob}\left\{S I N R^{T_{p}}(v)<E\left[S I N R_{i}\right]-x\right\} \\
& \sim C e^{-\mu\left(\frac{P_{i} G_{i} S_{T_{p}}}{E\left[S I N R_{i}\right]\left(\frac{E\left[S I N R_{i} S\right]}{x}-1\right)}\right)^{\nu}}
\end{aligned}
$$

which decays slowly with $T$ for data users with $0<\nu<$ 1. Therefore, it is expected that the outage probability is generally larger than that of the voice users. In particular, when $x \ll E\left[S I N R_{i}\right]$, Eq. (11) approximates to a Weibull bound as

$$
P r_{\text {out }} \sim C e^{-\mu\left(\frac{P_{i} G_{i}}{E^{2}\left[S I N R_{i}\right]}\right)^{\nu}\left(x S_{T_{p}}\right)^{\nu}} .
$$

\section{Simulation Results}

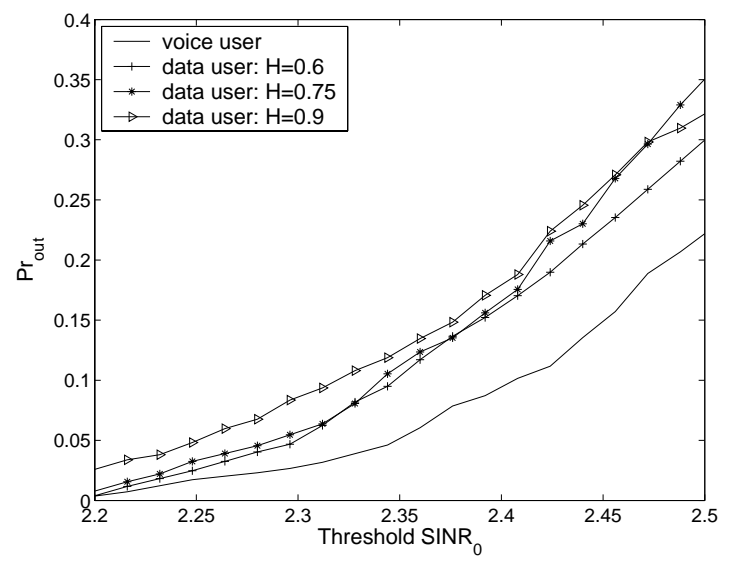

Fig. 4. Outage probability $\left(T_{p}=72 \mathrm{~ms}\right)$

Fig. 4 illustrates the outage probabilities for voice users and different data users with packet transmission time $T_{p} \simeq 72 \mathrm{~ms}$ (or with equivalent packet size of $576 \mathrm{~KB}$ ). We can see that, in general, data users have a higher outage probability than voice users. Among different data users, the larger the Hurst parameter $H$ in the LRD MAI, the higher the outage probability, which agrees with the trend predicted in our analysis results in Eq. (11).

Fig. 5 illustrates the outage probabilities for different packet transmission times, i.e., $5 \mathrm{~ms}, 72 \mathrm{~ms}, 130 \mathrm{~ms}$, $175 \mathrm{~ms}$ (which are corresponding to packet sizes of 40 bytes, 576 bytes, 1040 bytes and 1500 bytes). The outage probability for data users in Fig. 5(b) is larger than that for voice users in Fig. 5(a). It is also shown that increasing $T_{p}$ can reduce the outage probability for voice users, which indicates that better performance can be achieved if voice packets are transmitted in aggregation. However, the outage probabilities are similar for data users and have little dependence on the values of $T_{p}$. This is because the outage probabilities predicted by Eq. (11) for the above $T_{p}$ values have little difference due to a small $\nu=2-2 H=0.2$.

\section{Prediction on the Number of Active Users}

Although LRD data traffic tends to degrade the performance of a CDMA network, one may also harness long range dependency by designing protocols that can take advantage of the autocorrelation of an LRD process. More specifically, in a CDMA network dominated by LRD data traffic, the autocorrelation of MAI or SINR across many time scales can be used to predict the number of active users $K$ or MAI, which in turn can be used in rate control and Call Admission Control (CAC) to improve the network performance. In this section, we propose a Variable Period (VP) prediction and compare it with the prediction proposed in [4], [5], which we call Fixed Period (FP) prediction. 


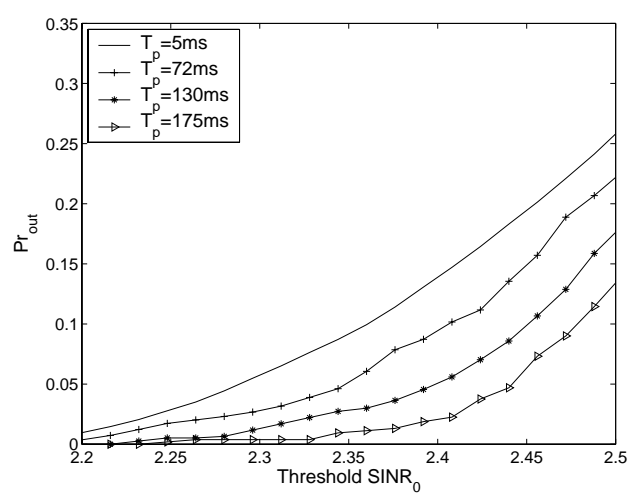

(a) voice users

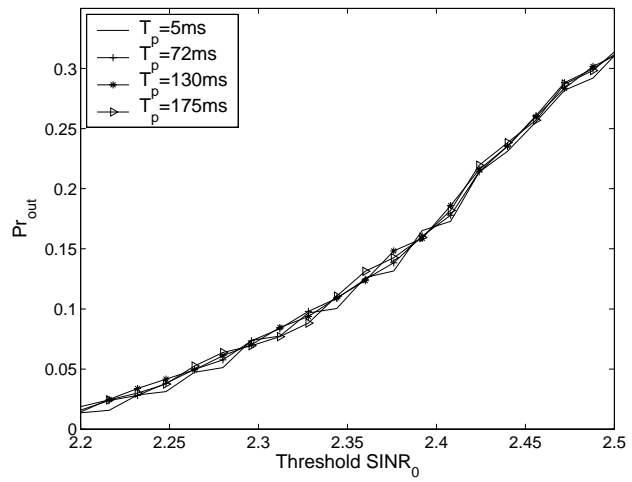

(b) data users, $\mathrm{H}=0.9$

Fig. 5. Outage probability

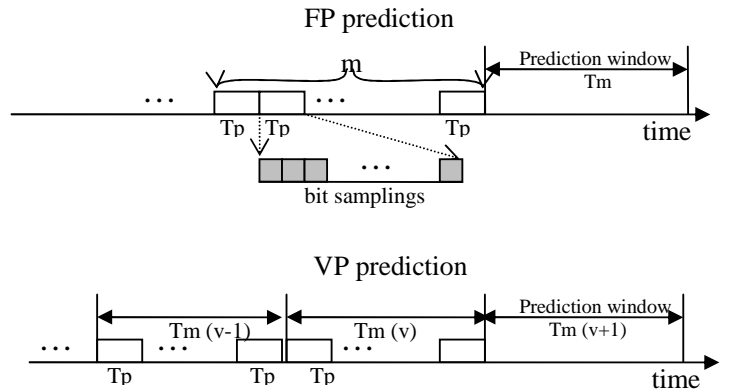

Fig. 6. FP vs. VP prediction

\section{A. Fixed Period Prediction}

We first review a multi-period MAI prediction scheme which we call Fixed Period (FP) prediction, which was proposed in [4], [5]. The FP scheme predicts the timescaled MAI, $I^{T_{m}}(v+1)$ in a prediction window $T_{m}$ based on a fixed number of measured (or historical) time-scaled MAI. Since $T_{p}$ is the typical time scale to calculate the outage probability as defined in Eq. (11), the time-scaled MAI measured in $T_{p}$ (with multiple bit samplings) is used to predict time-scaled MAI in $T_{m}$. A prediction window $T_{m}$ is usually larger than $T_{p}$, and $I^{T_{m}}$ is the average of multiple $I^{T_{p}}$ 's measured in $T_{m}$ (see Fig. 6), which is predicted as

$$
\hat{I}^{T_{m}}(v+1)=\frac{1}{m} \sum_{u=v m+1}^{(v+1) m} I^{T_{p}}(u) .
$$

Based on such a multi-period MAI prediction, the average number of active users in the $(v+1)$-th $T_{m}$ can also be predicted similarly as

$$
\hat{K}^{T_{m}}(v+1)=\frac{1}{m} \sum_{u=v m+1}^{(v+1) m} K^{T_{p}}(u),
$$

where $K^{T_{p}}(u)$ is the time-scaled $K$ during the transmission of the $u$-th packet. We call the above prediction for
$K$ or MAI as Fixed Period (FP) prediction because the number of historical values used for prediction is fixed as $m$ and each of the $m$ historical values contributes to the prediction with the same fixed weight $1 / \mathrm{m}$. Note that the system needs to store up to $m$ historical measured values for FP prediction.

\section{B. Variable Period Prediction}

In this subsection, we propose a new multi-period prediction which is called Variable Period (VP) prediction. VP predicts the next time-scaled value in $T_{m}$ based on all historical values available (not just $m$ historical values as in FP). Each historical value contributes to the prediction with an appropriate weight, i.e., usually a more recently measured value is associated with a larger weight. In addition, VP takes consideration of the autocorrelation in multiple time scales, i.e., autocorrelations in packet transmission time $T_{p}$ and in prediction window $T_{m}$. This is because the autocorrelation in multiple time scales for an LRD process is more significant than that for an SRD process. The VP prediction on $K$ has two steps as described next.

Suppose $K^{T_{p}}(v, j)$ is the time-scaled $K$ measured at the $j$-th $T_{p}$ of the $v$-th prediction window $T_{m}$. In VP prediction, we first calculate a weighted time-scaled $K$ based on $K^{T_{p}}(v, j)$ as

$$
\tilde{K}^{T_{p}}(v, j)=\left(1-\frac{1}{j^{\alpha}}\right) \tilde{K}^{T_{p}}(v, j-1)+\frac{1}{j^{\alpha}} K^{T_{p}}(v, j)
$$

and $\tilde{K}^{T_{p}}\left(v, \frac{T_{m}}{T_{p}}\right)$ is defined to be the weighted timescaled $K$ in the $v$-th $T_{m}$, which is the last value calculated from Eq. (15) when $j$ is set to $\frac{T_{m}}{T_{p}} \cdot K^{T_{m}}(v+1)$ is then predicted based on $\tilde{K}^{T_{p}}\left(v, \frac{T_{m}}{T_{p}}\right)$ and $\hat{K}^{T_{m}}(v)$ as

$$
\hat{K}^{T_{m}}(v+1)=\left(1-\frac{1}{v^{\beta}}\right) \hat{K}^{T_{m}}(v)+\frac{1}{v^{\beta}} \tilde{K}^{T_{p}}\left(v, \frac{T_{m}}{T_{p}}\right)
$$


where $\alpha$ and $\beta$ are constant parameters decided by the correlation coefficients between the predicted value and the measured values. With the above two-step prediction, time-scaled $K$ in both $T_{p}$ and $T_{m}$ are taken into consideration in the prediction. In addition, the weight of $K(v, j)$ is adjusted (decreased) in each prediction, and more recently measured $K(v, j)$ has a larger final weight in the prediction of $\hat{K}^{T_{m}}(v+1)$. Another advantage of VP prediction is that it is a recursive prediction, with which the system only needs to remember the latest calculated $\tilde{K}^{T_{p}}(v, j)$ and $\hat{K}^{T_{m}}(v)$. Therefore, VP consumes much less memory (which only needs $O(1)$ system memory) than FP (which has to store $m$ historical values and needs $O(m)$ system memory).

VP can also be extended to MAI prediction as well. The prediction on $K$ can be used in rate control and CAC to enhance the network performance as to be shown later in this paper.

\section{Simulation Results}

In this subsection, we evaluate the prediction accuracy of the proposed VP for data and voice users, respectively, and also compare VP with other existing schemes. In this simulation, we use the same setting as that described in Section III-A having 250 ON/OFF users with average activity factor of 0.4 (please refer to that section for more details). The Gaussian noise in the system is assumed to be $1 / 10$ of the transmitted signal strength, i.e., $N_{0}=$ $0.1 P$.

To evaluate the performance of VP, we use a similar prediction accuracy formula as Eq. (26) in [4]:

$$
\text { accuracy }=1-\frac{1}{N_{p}} \sum_{v=1}^{N_{p}} \frac{|\hat{K(v)}-K(v)|}{K(v)}
$$

where $N_{p}$ is the total number of predictions carried out in the simulation.

Fig. 7 illustrates the accuracy of FP, VP and the prediction based on the long term average value (shown as "EP" in the figure). We can see that EP has higher accuracy for voice users than that for data users (which are associated with a Hurst parameter of 0.9 in the simulation). This is because the average number of active voice users in $T_{m}$ (or $K^{T_{m}}$ ) approaches its long term mean value $E[K]$ quickly as $T_{m}$ increases with a smaller variance than that of data users. With a reasonable $T_{m}$ between 2 and 10 seconds $^{2}$, FP and VP have higher prediction accuracy than EP for data users only, and in particular, VP generally has higher accuracy than FP.

\footnotetext{
${ }^{2}$ The predict window $T_{m}$ in [4], [5] is 2 or 3 seconds
}

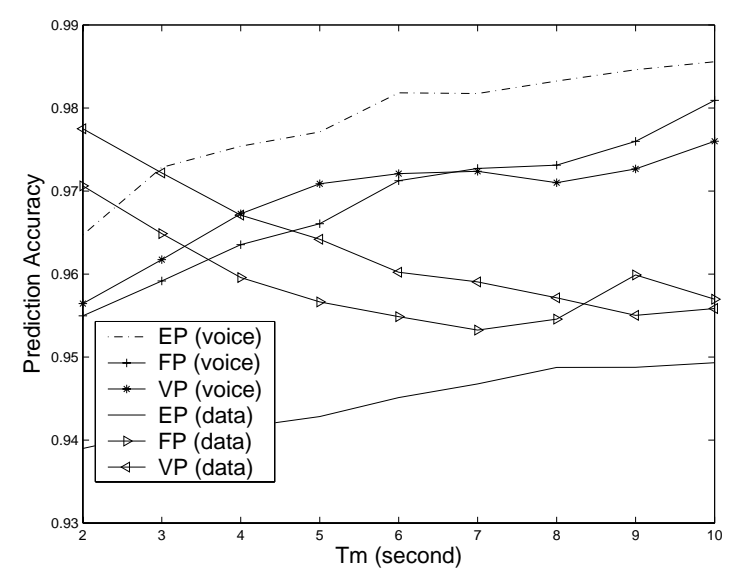

Fig. 7. Prediction accuracy

This is due to the fact that data users have LRD $K$, whose strong autocorrelation facilitates the prediction in FP and VP, and in particular, VP makes the best use of the autocorrelation crossing multiple time scales with appropriate weight assignments.

\section{RATE CONTROL}

In this section, we describe how rate control works with the proposed VP, and evaluate the outage and throughput performance of VP, FP, and EP respectively, when used in conjunction with rate control and compare the performances of CDMA systems with data users and voice users, respectively.

Rate control is used for interference management to achieve the optimal performance among existing users in a CDMA system. The base station sends rate control signals to mobile users according to its predicted MAI in window $T_{m}$. If the predicted MAI is weak, users can increase their transmission rates to achieve a higher throughput; Otherwise if the predicted MAI is strong, users should decrease their transmission rates to avoid high outage probability.

In order to achieve a target average user throughput and a target outage probability, we assume that a CDMA system can tolerate an average interference level measured at a time window $T_{m}$ up to $I_{0}^{T_{m}}$

$$
I_{0}^{T_{m}}=\frac{1}{S_{T_{m}}} P_{m} \sum_{u=t}^{t+S_{T_{m}}} \sum_{j=1}^{j=N} X_{j}(u) \frac{R_{j}}{R_{m}}=P_{m} K_{m}^{T_{m}}
$$

where $R_{m}$ is the maximum rate supported in the upper link and $P_{m}$ is the corresponding transmission power. In other words, the (equivalent) number of active users (transmitting at maximum rate $R_{m}$ ) that can be supported by the system at a time window $T_{m}$ is $K_{m}^{T_{m}}$. In the rate control of the CDMA system, we adjust the rate of each 
user according to the predicted $K^{T_{m}}$ using the following criteria:

1) If $\hat{K}^{T_{m}}(v+1)<K_{m}^{T_{m}}-\gamma_{u}$, then increase the transmission rate of each user $j$ with

$$
\Delta R_{j}=\frac{\left[K_{m}^{T_{m}}-\gamma_{u}-\hat{K}^{T_{m}}(v+1)\right]}{\hat{K}^{T_{m}}(v+1)} R_{j}
$$

2) If $\hat{K}^{T_{m}}(v+1)>K_{m}^{T_{m}}+\gamma_{l}$, then decrease the transmission rate of each user $j$ with

$$
\Delta R_{j}=\frac{\left[\hat{K}^{T_{m}}(v+1)-K_{m}^{T_{m}}-\gamma_{l}\right]}{\hat{K}(v+1)} R_{j}
$$

Here both $\gamma_{l}$ and $\gamma_{u}$ are constant thresholds.

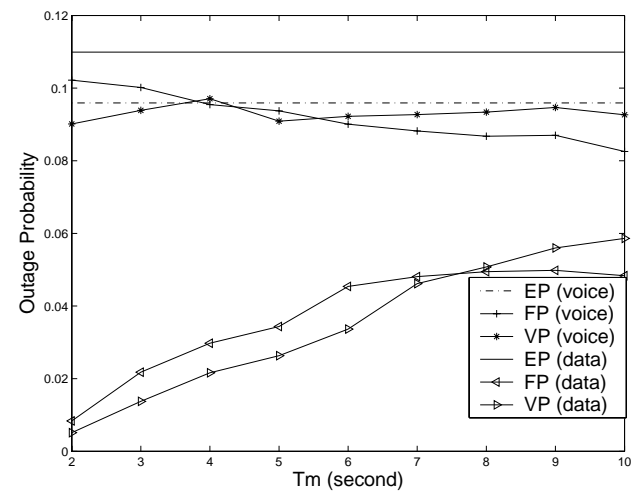

Fig. 8. Outage probability with rate control

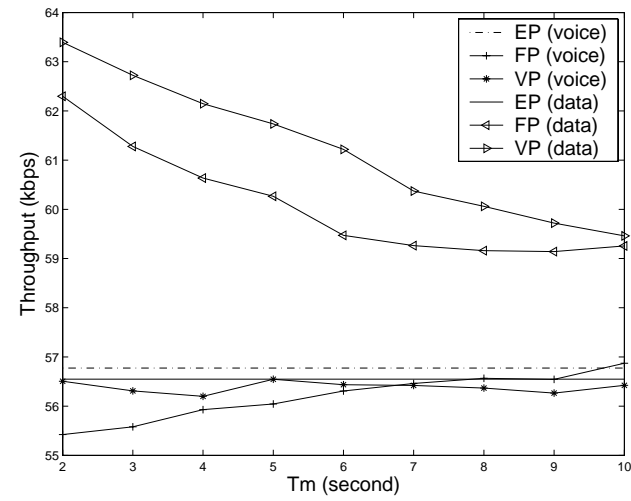

Fig. 9. Average user throughput with rate control

The simulation results are shown in Fig. 8 and 9 in terms of outage probability and average user throughput (for active users). The throughput for a user $i$ is defined as

$$
\hat{B}_{i}=\frac{1}{N_{s i m}} \sum_{v=0}^{N_{\text {Sim }}} \operatorname{sgn}\left(\operatorname{SINR} R^{T_{p}}(v)_{i}-S I N R_{0}\right) R_{i},
$$

where $N_{\text {sim }}$ is the total number of packets transmitted by user $i$ during its $\mathrm{ON}$ period throughout the simulation, and $\operatorname{sgn}($.$) is a function defined as$

$$
\operatorname{sgn}(x)=\left\{\begin{array}{l}
1, \quad \text { when } x>0 \\
0, \quad \text { otherwise }
\end{array}\right.
$$

In the simulation, a system with rate control based on EP is equivalent to that without rate control. We can see that rate control based on both FP and VP schemes can enhance the performance greatly for data users. In particular, VP outperforms FP with higher average user throughput and lower outage probability.

\section{CAll Admission Control}

In this section, we describe how call admission control (CAC) works with the proposed VP, and evaluate the outage and throughput performance of VP, FP, and EP respectively, when used in conjunction with $\mathrm{CAC}$ and compare the performances of CDMA systems with data users and voice users, respectively.

For a CDMA system with voice users, CAC is usually based on the average network capacity, or the average number of users $M_{0}$ that the system could support given a minimum SINR threshold $S I N R_{0}$ or the maximum number of active users $K_{0}$ [1], [15], i.e.,

$$
K_{0}=1+\frac{G}{S I N R_{0}}-\frac{W N_{0}}{P}
$$

and $M_{0}$ is calculated based on $K_{0}$ as:

$M_{0}=1+\frac{1}{E[X]}\left(K_{0}-1\right)=1+\frac{1}{E[X]}\left(\frac{G}{S I N R_{0}}-\frac{W N_{0}}{P}\right)$

where $E[X]$ is the average activity indicator of the users. In a CAC without prediction, new users are admitted if $N<M_{0}$, or equivalently if $E[K]<K 0$. Such a CAC is equivalent to the $\mathrm{CAC}$ that always predicts the future number of active users $K$ as $E[K]$. This is valid for voice users whose $K^{T_{m}}$ approaches $E[K]$ quickly with a relatively small variance. However, $K^{T_{m}}$ still has a relatively large variance, and predicting $K$ as $E[K]$ is not accurate for data users. For example, if a system has a total number of users $N<M_{0}$ but the number of active users $K^{T_{m}}>K_{0}$, admitting a new user could degrade the SINR of the new user as well as existing users.

In this section, we propose a CAC based on an extended VP prediction for data users as shown in Fig. 10. Our CAC uses a predicted average $K$ in a sliding window $\tau$, which is equivalent to average user life time (e.g., 5 minutes), to predict the average SINR of a new user. A new user is admitted into the system if the predicted average SINR is larger than the minimum threshold $S I N R_{0}$, or equivalently if the predicted $K$ is less than the maximum $K_{0}$, i.e., $\hat{K}^{\tau}<K_{0}$. Otherwise, the request 


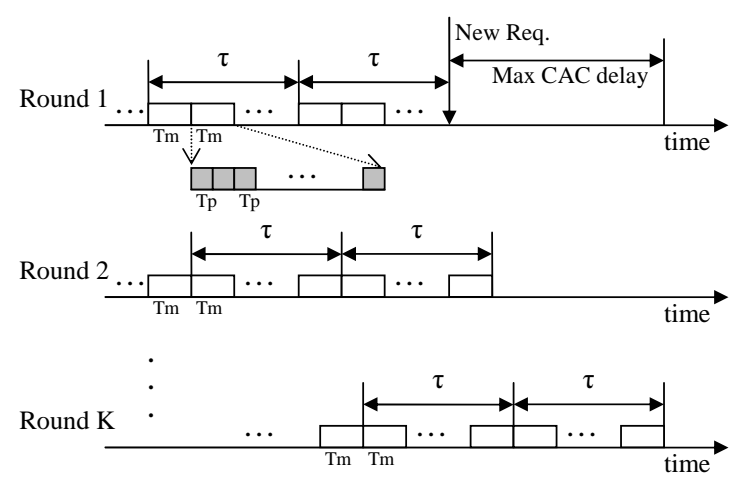

Fig. 10. Call admission for data users based on VP

will be buffered and the prediction is performed again in the next sliding window after a $T_{m}$ interval. If the new user cannot be admitted within a given maximum CAC delay, then it will be rejected.

With a CAC based on FP, the performance (in terms of theoretical throughput calculated based on $S I N R^{T_{p}}$ in [5]) of the new user is predicted based on the measured MAI (or $K$ ) in the $\tau$ just prior to making admission decisions. However, as $K$ is an LRD process exhibiting autocorrelation across multiple time scales, the calculation based on measurements in one window $\tau$ may not be sufficient. In this section, we extend our VP prediction proposed earlier in Section IV with a third step prediction for CAC, i.e., prediction in time scale $\tau$ as

$$
\left.\hat{K}^{\tau}(w+1)=\left(1-\frac{1}{w^{\gamma}}\right) \hat{K}^{\tau}(w)+\frac{1}{w^{\gamma}} \hat{K}^{T_{m}}\left(w, \frac{\tau}{T_{m}}\right)\right),
$$

where $K^{\hat{T}_{m}}\left(w, \frac{\tau}{T_{m}}\right)$ is the last predicted $K^{T_{m}}$ in the $w$ th $\tau$, and $\gamma$ is a constant decided by the autocorrelation in $\hat{K}^{\tau}$. With the extended VP, the prediction uses the measured $K$ in all previous sliding windows instead of using just one sliding window as in FP.

\section{A. Simulation Results}

In this section, we compare the average active user throughput defined in Eq. (19) and outage probability of the CDMA system with CAC based on the three prediction schemes, i.e., EP, FP and VP. Note that for a CAC based on EP, the system always keeps $N=M_{0}=$ 250 users in the system regardless of their activity status.

Figs. 11 and 12 illustrate the outage probability and average user throughput of the CDMA system with CAC based on different prediction schemes. CAC based on EP always has lower average outage probability and higher throughput for voice users than that for data users. This is because time-scaled $K$ for voice users has a smaller variance. Therefore, voice users have a higher prediction

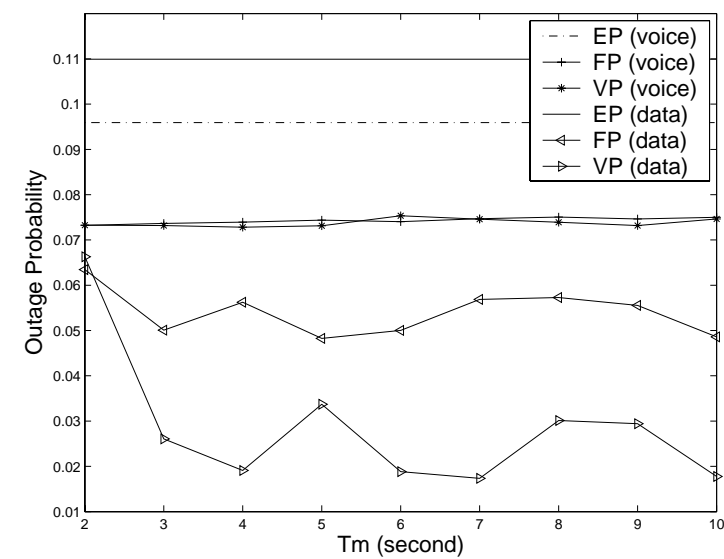

Fig. 11. Outage probability with CAC

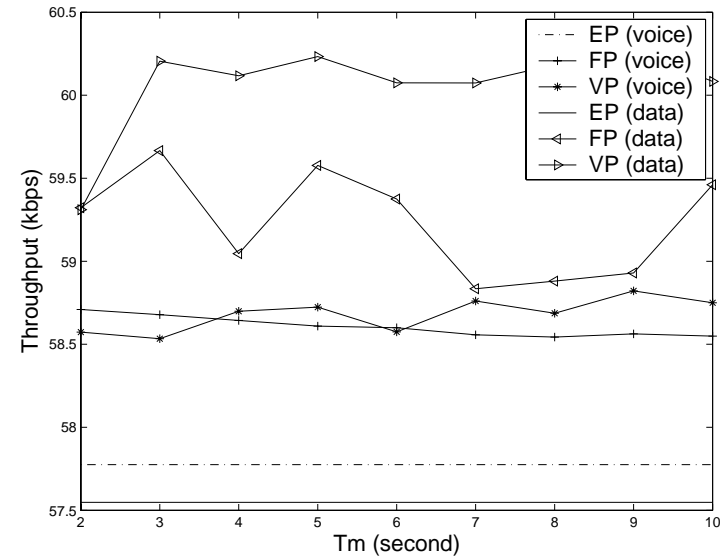

Fig. 12. Average user throughput with CAC

accuracy than data users when predicting $K^{T_{p}}$ with its mean value $E[K]$. However, CAC based on FP or VP enhances throughput and reduces outage probability for both data users and voice users. Specifically, for data users, CAC based on VP achieves higher throughput and lower outage probability as it can predict time-scaled $K$ more accurately than FP.

\section{Conclusions}

In this paper, we have studied the impact of long range dependency on the performance of a CDMA network including MAI, SINR and outage probability. It has been demonstrated through analysis and simulations that the time-scaled MAI and SINR in a CDMA system with data users have the same Gaussian or Gaussianlike distributions as those with voice users, but with slower decaying tail distributions and larger variances. Accordingly, the outage probability is higher in a CDMA system with data users. To harness the autocorrelation of MAI and SINR in a CDMA system with data users, a variable period (VP) prediction has also been proposed for such a CDMA system according to the fact that 
the number of active users or MAI has autocorrelations across multiple time scales. The performance of VP has been compared with existing schemes including fixed period prediction proposed in [4], and VP prediction has been demonstrated to be more accurate. We have also applied VP prediction to rate control and call admission control and shown that better system performance in terms of lower outage probability and higher average user throughput can be achieved using the proposed scheme.

\section{REFERENCES}

[1] Kamil Sh. Zigangirov, "Theory of code division multiple access communication," in IEEE series on Digital and Mobile Communication, 2004.

[2] V. Paxson and A. Floyd, "Wide area traffic: The failure of poisson modeling," IEEE/ACM Transactions on Networking, vol. 3, no. 3, pp. 226-244, 1995.

[3] X. Yu, I. Thng, Y. Jiang, and C. Qiao, "Upper bounds on individual queue length distribution in gps and pgps with lrd traffic input," IEEE/ACM Transactions on Networking, vol. 13, no. 3, June 2005.

[4] Junshan Zhang, Ming Hu, and Ness B. Shroff, "Bursty data over CDMA: MAI self similarity, rate control and admission control," in Proceedings of INFOCOM, 2002.

[5] J. Zhang, M. Hu, and N. B. Shroff, "Bursty data over cdma: Predictive mai temporal structure, rate control and admission control," IEEE Computer Networks, vol. 42, no. 6, pp. 779996, 2003.

[6] W. Willinger, M. Taqqu, W. Leland, and D. Wilson, "Selfsimilarity in high-speed packet traffic: Analysis and modeling of ethernet traffic measurements," Statistical Science, vol. 10, pp. 67-85, 1995.

[7] W. Leland, M. Taqqu, W. Willinger, and D. Wilson, "On the self-similar nature of ethernet traffic (extended version)," IEEE/ACM Transactions on Networking, vol. 2, no. 1, pp. 115, 1994.

[8] R. Addie, M. Zukerman, and T. Neame, "Broadband traffic modeling: Simple solutions to hard problems," IEEE Communications Magazine, vol. 36, no. 8, pp. 88-95, 1998.

[9] W. Willinger, V. Paxson, and M. Taqqu, "Self-similarity and heavy tails: Structural modeling of network traffic," A Practical Guide to Heavy Tails: Statistical Techniques and Applications, vol. 23, pp. 27-53, 1998.

[10] D. Heath, S. Resnick, and G. Samorodnitsky, "Heavy tails and long range dependence in on/off processes and associated fluid models," Math. Oper. Res., vol. 23, pp. 145-165, 1998.

[11] R. Yates, "A framework for uplink power control in cellular radio systems," IEEE Journal on Selected Areas in Communications, vol. 13, no. 7, pp. 1341-1347, 1995.

[12] J. Zander, "Performance of optimum transmission power control in celluar radio systems," IEEE Transaction on Vehicular Technology, vol. 41, no. 1, pp. 57-62, 1992.

[13] B. B. Mandelbrot, "Long-run linearity, locally gaussian processes, h-spectra and infinite variances," Intern. Econom. Rev., vol. 10, pp. 82-113, 1969.

[14] S. U. Pillai A. Papoulis, "Probability random variables, and stochastic processes," McGraw-Hill, 4th edition, 2001.

[15] T. S. Rappaport, "Wireless communications: Principles and practice," in Prentice Hall, 1996.

\section{APPENDIX}

In this appendix, we prove that the time-scaled SINR is approximately "Gaussian-like" distributed for a CDMA system with either data users or voice users. And we also prove that the SINR is an LRD process and its corresponding time-scaled process has slow-decaying tail distributions.

With power control, SINR for user $i$ at the $u$ th sampling in Eq. (8) is rewritten as

$$
\begin{aligned}
\operatorname{SINR}_{i}(u) & =\frac{G_{i}}{N_{0}(u) W / P_{i}+\sum_{j=1, j \neq i}^{N} X_{j}(u) \frac{R_{j}}{R_{i}}} \\
& =\frac{G_{i}}{N_{0}(u) W / P_{i}+K_{i}(u)}
\end{aligned}
$$

where $G_{i}=W / R_{i}$ is the processing gain for user $i$. The autocorrelation of $S I N R_{i}$ is then defined as

$$
\begin{aligned}
& r_{S I N R}(m) \\
& =E\left[\frac{G_{i}}{\frac{N_{0}(u) W}{P_{i}}+K_{i}(u)} \times \frac{G_{i}}{\frac{N_{0}(u+m) W}{P_{i}}+K_{i}(u+m)}\right] \\
& =\frac{G_{i}^{2}}{\frac{E\left[N_{0}\right]^{2} W^{2}}{P_{i}^{2}}+\frac{2 E[K] E\left[N_{0}\right] W}{P_{i}}+E\left[K_{i}(u) K_{i}(u+m)\right]} \\
& =\frac{G_{i}^{2}}{\frac{E\left[N_{0}\right]^{2} W^{2}}{P_{i}^{2}}+\frac{2 E[K] E\left[N_{0}\right] W}{P_{i}}+r_{K}(m)}
\end{aligned}
$$

Assume that $N_{0}^{T}(v)$ and $K_{i}^{T}(v)$ are time-scaled noise and time-scaled number of active users. The average SINR in time scale $T$ is calculated based on the values of $N_{0}^{T}(v)$ and $K_{i}^{T}(v)$ as

$$
\operatorname{SINR}_{i}^{T}(v)=\frac{G_{i}}{N_{0}^{T}(v) W / P_{i}+K_{i}^{T}(v)}
$$

which is called (equivalent) time-scaled ${ }^{3}$ SINR. Its autocorrelation is

$$
\begin{aligned}
& r_{S I N R_{i}}^{T}(m)=E\left[S I N R_{i}^{T}(v) S I N R_{i}^{T}(v+m)\right] \\
= & E\left[\frac{G_{i}}{\frac{N_{0}^{T}(v) W}{P_{i}}+K_{i}^{T}(v)} \times \frac{G_{i}}{\frac{N_{0}^{T}(v+m) W}{P_{i}}+K_{i}^{T}(v+m)}\right] \\
= & \frac{G_{i}^{2}}{\frac{E\left[N_{0}\right]^{2} W^{2}}{P_{i}^{2}}+\frac{2 E\left[N_{0}\right] E[K] W}{P_{i}}+E\left[K_{i}^{T}(v) K_{i}^{T}(v+m)\right]} \\
= & \frac{G_{i}^{2}}{\frac{E\left[N_{0}\right]^{2} W^{2}}{P_{i}^{2}}+\frac{2 E\left[N_{0}\right] E[K] W}{P_{i}}+r_{K}^{T}(m)}
\end{aligned}
$$

Since $K$ is an LRD process, we have $r_{K}^{T}(m)=r_{K}(m)$. Therefore, the above Eq. (26) is the same as Eq. (24), i.e., $r_{S I N R}^{T}(m)=r_{S I N R}(m)$. Therefore, SINR is analogously an LRD process because its time-scaled process has similar autocorrelation as its instantaneous process (as an LRD process does), but with a slight different definition of time-scaled SINR from Eq. (1).

\footnotetext{
${ }^{3}$ Note that here the (equivalent) time-scaled SINR is different from the definition of time-scaled process in Eq. (1)
} 
The PDF of $S I N R_{i}^{T}$ is:

$$
\begin{aligned}
f_{S I N R_{i}}^{T}(z) & =\operatorname{Pr}\left\{\frac{G_{i}}{N_{0}^{T} W / P_{i}+K_{i}^{T}}=z\right\} \\
& =\operatorname{Pr}\left\{\frac{1}{\frac{K_{i}^{T}}{G_{i}}+\frac{N_{0}^{T} W}{G_{i} P_{i}}}=z\right\}
\end{aligned}
$$

Suppose that $n(u)$ is the Gaussian noise amplitude from one sampling, which follows a normal distribution of $N\left(0, \sigma_{n}^{2}\right)$. Then $N_{0}(u)=n^{2}(u)$ is the noise power per frequency obtained from that sampling, which follows an Chi-Square distribution, i.e.,

$$
f_{n^{2}}(x)=\frac{1}{\sqrt{2 \pi x} \sigma_{n}} e^{-\frac{x}{2 \sigma_{n}^{2}}}
$$

with mean $E\left[N_{0}\right]=\sigma_{n}^{2}$ and variance $\operatorname{Var}\left[N_{0}\right]=2 \sigma_{n}^{4}$. Let $N_{0}^{\prime T}=\frac{N_{0}^{T} W}{G_{i} P_{i}}$ with PDF $f_{N_{0}^{\prime}}^{T}(x)$ and let $K_{i}^{\prime T}=\frac{K_{i}^{T}}{G_{i}}$ with PDF $f_{K^{\prime}}^{T}(x)$. Suppose function $h^{T}\left(K_{i}^{T}, N_{0}^{T}\right)=\frac{K_{i}^{T}}{G_{i}}+\frac{N_{0}^{T} W}{G_{i} P_{i}}=$ $K_{i}^{\prime T}+N_{0}^{\prime T}$ has a PDF $f_{h}^{T}(x)$, which is the convolution of $f_{K_{i}^{\prime}}^{T}(x)$ and $f_{N_{0}^{\prime}}^{T}(x)$, i.e.,

$$
f_{h}^{T}(x)=\int_{-\infty}^{\infty} f_{K_{i}^{\prime}}^{T}(u) f_{N_{0}^{\prime}}^{T}(x-u) d u
$$

then the PDF of $S I N R_{i}^{T}$ is expressed as the following Eq. (30) [14]:

$$
f_{S I N R_{i}}^{T}(z)=\frac{1}{|z|} f_{h}^{T}\left(\frac{1}{z}\right)
$$

Eq. (30) applies to both instantaneous SINR and time-scaled SINR. To calculate the PDF of instantaneous SINR, we first obtain the PDF of instantaneous $K_{i}^{\prime}$ and $N_{0}^{\prime}$ as

$$
\begin{gathered}
f_{K_{i}^{\prime}}(x)=f_{K_{i}}\left(G_{i} x\right) \times G_{i}=\frac{G_{i}}{\sqrt{2 \pi \sigma_{K}^{2}}} e^{-\frac{\left(G_{i} x-E\left[K_{i}\right]\right)^{2}}{2 \sigma_{K}^{2}}} \\
f_{N_{0}^{\prime}}(x)=f_{n^{2}}\left(\frac{G_{i} P_{i} x}{W}\right) \times \frac{G_{i} P_{i}}{W}=\frac{\sqrt{G_{i} P_{i}}}{\sqrt{2 \pi W x} \sigma_{n}} e^{-\frac{G_{i} P_{i} x}{2 W \sigma_{n}^{2}}}
\end{gathered}
$$

$f_{S I N R_{i}}(z)$ is then rewritten from Eq. (30) as:

$$
\begin{aligned}
& f_{S I N R_{i}}(z)=\frac{1}{|z|} \int_{-\infty}^{\infty} \frac{G_{i}}{\sqrt{2 \pi \sigma_{K}^{2}}} e^{-\frac{\left(G_{i} u-E\left[K_{i}\right]\right)^{2}}{2 \sigma_{K}^{2}}} \\
& \times \frac{\sqrt{G_{i} P_{i}}}{\sqrt{2 \pi W\left(\frac{1}{z}-u\right)} \sigma_{n}} e^{-\frac{G_{i} P_{i}\left(\frac{1}{z}-u\right)}{2 W \sigma_{n}^{2}}} d u
\end{aligned}
$$

To calculate the PDF of time-scaled SINR from Eq. (30), we need to obtain the distribution of both $N_{0}^{\prime T}$ and $K^{\prime T}$. Since the white Gaussian noise is an independent process, as $T$ increases, $N_{0}^{\prime T}$ approaches Gaussian distribution according to the central limit theorem with mean $E\left[N_{0}^{T}\right]=W \sigma_{n}^{2} / P_{i} G_{i}$ and variance $\operatorname{Var}\left[N_{0}^{\prime T}\right]=2 W^{2} \sigma_{n}^{4} / P_{i}^{2} G_{i}^{2} S_{T}$. While $K_{i}^{\prime T}$ follows a Gaussian distribution with mean $E\left[K_{i}^{\prime T}\right]=E[K] / G_{i}$ and variance $\operatorname{Var}\left[K_{i}^{\prime T}\right]=\sigma_{K}^{2} / G_{i}^{2} S_{T}^{2-2 H}$. Similar to $I_{i}^{T}$, the variance of $K_{i}^{\prime T}$ is the same as that predicted by the central limit theorem only when $K_{i}$ is SRD with voice users (i.e., $H=0.5$ ); For data users (i.e., $0.5<H<1$ ), the variance is larger than that predicted by the central limit theorem. Since both $K_{i}^{\prime T}$ and $N_{0}^{\prime T}$ follow Gaussian distributions, and also because $K_{i}^{\prime T}$ and $N_{0}^{\prime T}$ are independent of each other, the sum of the $K_{i}^{\prime T}$ and $N_{0}^{\prime T}$ still follows a Gaussian distribution with mean

$$
E\left[h^{T}\right]=E\left[K_{i}^{\prime T}\right]+E\left[N_{0}^{\prime T}\right]=\frac{E[K]}{G_{i}}-\frac{\sigma_{n}^{2} W}{G_{i} P_{i}}
$$

and variance

$$
\begin{aligned}
\operatorname{Var}\left[h^{T}\right] & =\operatorname{Var}\left[K_{i}^{\prime T}\right]+\operatorname{Var}\left[N_{0}^{\prime T}\right] \\
& =\frac{\sigma_{K}^{2}}{G_{i}^{2} S_{T}{ }^{2-2 H}}+\frac{2 W^{2} \sigma_{n}^{4}}{G_{i}^{2} P_{i}^{2} S_{T}}
\end{aligned}
$$

Therefore, the PDF of $S I N R_{i}^{T}$ in Eq. (30) can be approximated as:

$$
f_{S I N R_{i}}^{T}(z) \simeq \frac{1}{\sqrt{2 \pi \operatorname{Var}\left[h^{T}\right]}|z|} e^{-\frac{\left(\frac{1}{z}-E\left[h^{T}\right]\right)^{2}}{2 \operatorname{Var}\left[h^{T}\right]}}
$$

which we call as a "Gaussian like" distribution. Note that for voice users, the impact of $T$ on the variance shown in Eq. (35) is more significant than that for data users. And in general, the PDF of $S I N R_{i}^{T}$ in Eq. (36) has a larger variance for data users than that for voice users for any $S_{T}>1$.

Now we exam the tail distribution of $S I N R^{T}$, i.e., $\operatorname{Pr}\left\{S I N R_{i}^{T}>E\left[S I N R_{i}\right]+x\right\}$, where $x$ is the tail of $S I N R^{T}$. Since Gaussian noise is an independent process, its tail distribution decays quickly with $T$. With some large finite time scale $T$, the tail probability of $N_{0}^{T}$ can be ignored in the tail distribution of $S I N R^{T}$, and the parameter $N_{0}^{T}$ in $S I N R^{T}$ is approximated with its mean value $\sigma_{n}^{2}$ as,

$$
\begin{aligned}
& \operatorname{Pr}\left\{S I N R_{i}^{T}(v)>E\left[S I N R_{i}\right]+x\right\} \\
& =\operatorname{Pr}\left\{\frac{G_{i}}{\sigma_{n}^{2} W / P_{i}+K_{i}^{T}(v)}>E\left[S I N R_{i}\right]+x\right\} \\
& =\operatorname{Pr}\left\{K_{i}^{T}(v)<\frac{G_{i}}{E\left[S I N R_{i}\right]+x}-\frac{\sigma_{n}^{2} W}{P_{i}}\right\} \\
& =\operatorname{Pr}\left\{I_{i}^{T}(v)<\left(\frac{P_{i} G_{i}}{E\left[S I N R_{i}\right]}-\sigma_{n}^{2} W\right)\right. \\
& \left.-\frac{P_{i} G_{i} x}{E\left[S I N R_{i}\right]\left(E\left[S I N R_{i}\right]+x\right)}\right\} \\
& =\operatorname{Pr}\left\{I_{i}^{T}(v)>E\left[I_{i}\right]+\frac{P_{i} G_{i} x}{E\left[S I N R_{i}\right]\left(E\left[S I N R_{i}\right]+x\right)}\right\}
\end{aligned}
$$

The last step of Eq. (37) is because $I_{i}^{T}$ has a symmetric Gaussian distribution centered at $E\left[I_{i}\right]$.

Since it has been proved that $I_{i}$ is an WBB LRD process, Eq. (37) can be simplified as follows

$$
\begin{aligned}
& \operatorname{Pr}\left\{S I N R_{i}^{T}>E\left[S I N R_{i}^{T}\right]+x\right\} \sim \\
& \quad C e^{-\mu\left(\frac{P_{i} G_{i} S_{T}}{E\left[S I N R_{i}^{T}\right]\left(1+\frac{E\left[S I N R_{i}^{T}\right]}{x}\right)}\right)^{\nu}}
\end{aligned}
$$

With $\nu<1$ for data users, the tail distribution in Eq. (38) decays slow with $T$, and the tail probability is larger than that of the time-scaled SINR with voice users $(\nu=1)$. In particular, if $x \ll E\left[S I N R_{i}\right]$, Eq. (38) approximates a Weibull bound as in (10). 